\title{
Groin and hip quandaries
}

\author{
H Millson \\ Sports Physiotherapy, Orchard View, School Road, Coddenham, Suffolk, UK \\ H Millson, MPhil Sports Physio, MCSP
}

Corresponding author: H Millson (helen.millson@iprsgroup.com)

\begin{abstract}
There is little consensus on the diagnosis, pathophysiology, investigation and management of groin injuries. A key factor in making the correct diagnosis is to firstly understand the anatomy and likely generators of pain in the region. This requires an understanding of the two joints in the pelvis - the hip joint and the pubic symphysis - which are at the centre of many movements. There are a multitude of varying studies on this topic. However, most importantly, many of the groin/hip pathologies can be averted by thorough and specific prehabilitation, bearing in mind the entire kinetic chain and addressing total function above and below the pelvis.
\end{abstract}

S Afr J SM 2012;24(4):127-128. DOI:10.7196/SAJSM.359

The groin, an anatomical region where diagnosis and symptoms are often confusing, may also represent a 'Bermuda triangle' for clinicians to disappear in vortices of suppositions and assumptions. The wellknown FIFA sports physiotherapist, Dr Mario Bizzini, called groin pain 'The Bermuda triangle of sports medicine?' - with good reason. ${ }^{1}$

Why is there so little international consensus on diagnosis, pathophysiology, investigation and management? Is this because the diagnosis is multifactorial and there are 82 differential diagnoses relating to the groin, as well as another 41 related to the hip? It is not simple.

In elite sports it is often very difficult for a doctor to diagnose accurately and thus be able to send the player to the relevant specialist who deals only with a specific area. There are anecdotal examples, such as that of a top professional sportsman who has had four different specialist opinions and four different surgical procedures.

One needs sound clinical judgement for the overall picture. One of the key points is to understand the entire anatomy and the likely generator of pain. The two joints in the pelvis should be emphasised - the hip joint and the pubic symphysis - which are at the centre of many movements. The term osteitis pubis (OP) to describe groin pathology ${ }^{2}$ is now used with caution, because no clinical parameter develops in isolation. ${ }^{3}$ Also, an MRI scan showing bone marrow oedema in the symphysis pubis does not always correlate with the clinical symptoms. This is often the case in professional footballers where asymptomatic $\mathrm{OP}$ is fairly common.

Studies suggest that the radiological consequence of bone marrow oedema in sport represents load - nothing more - and is part of mechanotransduction adaptation of the osteocytes in the bone in response to increased activity. ${ }^{4}$ Radiology needs to be balanced with the physical examination to determine the true nature of the findings and at no stage should be used as a definitive diagnosis.

Garvey et al. state that it is most important to deal firstly with the pubic joint and its relevant attachments, although one should always bear in mind the associated structures around the pelvis and the entire kinetic chain. ${ }^{5}$ Brukner and Kahn emphasise that the increased load on the pelvis should be identified and reduced as a matter of course. ${ }^{6}$ It would be most useful if studies could show whether the increased signal/presence of bone marrow oedema is a precursor to the development of groin pain.
An important question is whether clinicians really understand the importance of the adductor muscles. Adductor muscle injuries are present in $13-70 \%$ of groin injuries.

Adductor pain without strength loss is commonly associated with a hip condition. Adductor longus dysfunction may result in instability and anterior subluxation of the symphysis. Both entities are mechanically related and $\mathrm{OP}$ and adductor dysfunction frequently coexist. Adductor strength may be an important objective outcome for prevention and rehabilitation and to assess return-to-play readiness, as well as for identifying and reducing the sources of increased load on the pelvis.

It is also important to consider the other anatomical structures around the groin, including the ilio-psoas, ligamentum teres, greater trochanter area, gluteus medius and rectus abdominus. ${ }^{7}$ These anatomical structures may have to adapt to extraordinary biomechanics around the groin area and need to be addressed in prehabilitation/rehabilitation management. Further, the pelvic floor may need to be considered if all other signs of groin pain have been cleared. Experienced clinical judgement and clinical reasoning remain a critical element in the diagnostic pathway. There is no 'one-stopshop' to diagnose all.

Although it is generally believed that conservative management should be undertaken before surgery is considered, the time line differs with different specialists. There are some fundamental principles, including maximising the range of motion in the hips, regaining effective muscle strength without stressing the pubic symphysis, facilitating abdominals/ adductors as pubic symphysis stabilisers, and ensuring that a global strengthening programme is undertaken. It is most important to establish a benchmark and progress with objective functional and clinical markers that are monitored on an on-going basis. Postoperative rehabilitation programmes varied from 3 to 12 weeks.

In surgical management there are most differences of opinion and one should be sceptical of one-size-fits-all operations. There are a number of varied surgical opinions ranging from the time span for conservative treatment prior to surgery to the actual surgical procedure, and to the time line for return to play. With hernias, there is controversy regarding the prevalence of a true hernia. There is currently no consensus as to the ideal operating technique; surgical procedures are continuing to evolve and improve. 
The evolving interventions regarding hip pathology also need to be considered. A combination of hip and pelvic injuries occurs in about $14 \%$ of the population and yet the hip has not historically always been considered as part of this aetiology. Studies investigating physical tests for hips have been of poor quality, demonstrating a need for further research in this area. Negative test results are of more use in ruling out the condition, given the high reported sensitivities of many tests. There are many quandaries pertaining to hip pathology. Femoral acetabular impingement exists, but is not necessarily a diagnosis.

The surgeon must weigh the risks of performing a potentially unnecessary procedure in an asymptomatic individual with cam morphology against the risks of allowing unchecked progression of the deleterious process. One should also bear in mind that excessive removal of acetabular labrum and ilio-femoral ligament in surgery can be detrimental. ${ }^{8}$

A most important factor for the clinician is that preliminary studies have demonstrated that having a hip joint restriction precedes the development of chronic groin injury and may be a risk factor for this condition. Therefore, it may be most important to maintain range of motion in the management to prevent groin problems. Future research is required to determine the extent to which physiotherapy intervention aimed at improving hip kinematics would be effective in treating individuals with labral injuries.

In conclusion, it is most important to highlight the difference between an anatomical description and a functional description. Further long-term studies are required as well as trials where clinical entities are correlated with systemic investigation, including MRI and ultrasonography.

So what is the solution to this Bermuda triangle quandary?

- One needs to assess how the patient's functional movement influences both the hip and the pubic symphysis. What causes pain and where? Try to understand the entire kinetic chain with its related function to the pelvis/groin/hip. Then perform relevant clinical tests as well as sports-specific functional tests. Clinical reasoning must be at the forefront at all times.

- In spite of minimal evidence-based medicine in this regard, it seems that the most pertinent point is that many of the groin/hip pathologies can be averted by thorough and specific prehabilitation, bearing in mind the entire kinetic chain and addressing total function around the pelvis - above and below.

\section{References}

1. Bizzini M. The groin area: The Bermuda triangle of sports medicine? Br J Sports Med 2011;45(1):1. [http://dx.doi.org/10.1136/bjsm.2010.081828]

2. Verrall GM, Henry L, Fazzalari NL, Slavotinek JP, Oakeshott RD. Bone biopsy of the parasymphyseal pubic bone region in athletes with chronic groin injury demonstrates new woven bone formation consistent with a diagnosis of pubic bone stress injury. Am J Sports Med 2008;36(12):2425-2431.

3. Hölmich P. Long-standing groin pain in sportspeople falls into three primary patterns, a 'clinical entity' approach: A prospective study of 207 patients. Br J Sports Med 2007;41(4):247-252.

4. Paajanen H. 'Sports hernia' and osteitis pubis in an athlete. Duodecim 2009;125(3):261266.

5. Garvey JF, Read JW, Turner A. Sportsman hernia: What can we do? Hernia 2010;14(1):17-25.

6. Brukner P, Khan K. Clinical Sports Medicine. 4th ed. Australia: McGraw-Hill, 2012.

7. Philippon MJ, Decker MJ, Giphart JE, Torry MR, Wahoff MS, LaPrade RF. Rehabilitation exercise progression for the gluteus medius muscle with consideration for iliopsoas tendinitis: An in vivo electromyography study. Am J Sports Med 2011;39(8):1777-1785.

8. Abellàn J, Esparza F, Blanco A, et al. Radiological evidence of femoroacetabular impingement in asymptomatic athletes. Br J Sports Med 2011;45:333. [http://dx.doi. org/10.1136/bjsm.2011.084038.65]

A more comprehensive list of references can be obtained from the author on request. 